\title{
Role of Intrinsic Defects in Nanowires
}

\author{
Usha Philipose \\ Department of Physics, University of North Texas \\ United States of America
}

\section{Introduction}

The properties of nanowires are distinctively different from those of bulk materials because they are affected by quantum confinement effect and large surface-to-volume ratios. A fundamental understanding of the intrinsic properties of nanowires, in terms of their structure, electronic and optical properties as well as a study of the nanowire surface is required to develop them for future device applications. The morphology and properties of the nanowires are strongly dependent on the synthesis process and growth parameters. Key to realizing the full potential of nanowires is the ability to synthesize uniform, defect free nanowires. Any deviation from stoichiometry leads to imperfections within the nanowire crystal lattice and affects its properties. Efforts to optimize growth conditions are spearheaded by investigations to identify and control native point defects inherent in semiconductors. Structural characterization using electron microscopy is usually the first step towards identifying the quality of the nanowire in terms of composition, morphology and defects like twinning and stacking faults. Optical characterization tools like Photoluminescence (PL) spectroscopy is useful for extracting information on band-to-band transition and other sub-band electronic transitions, thus providing information on any deviations from stoichiometry. However, it cannot provide a comprehensive view of every aspect of the optical properties of nanowires. Hence, complementary experiments like time-resolved PL, pump probe measurements, cathodoluminescence, absorption and reflection spectroscopy are often used. In this chapter, we will present a brief review of some of the techniques used in identifying and characterizing defects in semiconductor nanowires. Studies drawn primarily from the author's work, addressing the role of intrinsic point defects in nanowires and discussion of strategies for determining the type, energy and concentration of defects in nanowires will be presented in this chapter.

The presence of low energy luminescence bands in un-intentionally doped nanowires is evidence that intrinsic point defects (IPDs) play a significant role in the emission spectrum. IPDs in a compound semiconductor are vacancies, interstitials and antisites. In bulk semiconductors, IPDs are often studied by Optical Detection of Magnetic Resonance (ODMR) and Electron Paramagnetic Resonance (EPR) studies. However, the use of such studies on nanowires is only recent (Mal et al., 2010). It is important to understand the behavior of IPDs for the successful application of any semiconductor, since they control, directly or indirectly, doping, compensation, minority carrier lifetime, and luminescence efficiency. Semiconductor doping, in particular, can be adversely affected by IPDs, by causing self-compensation. For example, IPDs which act as donors may compensate the deliberately introduced acceptors. In group II-VI semiconductors like $\mathrm{ZnO}$, specific native defects have long been believed to play 
a very important role. As-grown $\mathrm{ZnO}$, both in bulk and nanoscale, exhibits unintentional n-type conductivity, and its source is attributed to IPDs like oxygen and zinc vacancies as well as zinc interstitials. IPDs are also suspected to play an important role in the observed room temperature ferromagnetism in transition metal (TM) doped II-VI semiconductors through defect mediated coupling of the local d-electrons of the TM ions. There are also reports that nanoscale clusters of intrinsic $\mathrm{ZnO}$ defects containing ferromagnetically aligned spins are being confused with ferromagnetic Dilute Magnetic Semiconductors (Potzger \& Zhou, 2009). This chapter will provide a review of experimental studies of IPDs in II-VI semiconductors and their influence on the optical and electronic properties of semiconductor nanowires. A brief description of a model based on chemical thermodynamics will be used to review the thermodynamics of intrinsic defects in nanowires and to show the dependence of IPDs on growth parameters. Using structural and optical characterization techniques, studies revealing a correlation between growth parameters, structural defects like stacking faults and twinning defects as well as the role of IPDs in determining the properties of nanowires will be presented.

The surface of nanowires also presents significant challenges as well as opportunities for affecting nanowire properties. On account of the large surface to volume ratio in nanowires, their surfaces can dominate their quantum confined properties. Hence, it is necessary to elucidate the fundamental relationships between nanowire interfaces with the environment and their electronic and optical properties. We will present a brief review of the efficacy of various surface passivation schemes. This chapter will thus review scientific advances made in the study of fundamental intrinsic properties of nanowires, namely an estimation and determination of type of defects in nanowires, with an aim to develop nanowires for viable applications.

\section{Defect analysis using a thermodynamic model}

The defect analysis presented in this section has been developed for ZnSe nanowires grown by Chemical Vapor Deposition (CVD), but the general analysis can be extended to most semiconductor materials. ZnSe is an important material for fabrication of optoelectronic devices. This direct band gap material should be characterized by high luminescence efficiency, but despite decades of research, emission is often dominated by low-energy luminescence bands caused by point defects which introduce energy levels within the band gap of ZnSe. The principle defects in a binary compound like ZnSe include vacancies on the sub-lattice of $\mathrm{Zn}\left(V_{Z n}^{x}\right)$ and Se $\left(V_{S e}^{x}\right)$, interstitial atoms $\left(Z n_{i}\right)$ and $\left(S e_{i}\right)$, antistructural defects $\left(S e_{Z n}\right)$ and $\left(Z n_{S e}\right)$ (Se atom in the Zn sub-lattice and vice versa) and positively and negatively single- and double- charged vacancies $\left(V_{S e}^{+}, V_{S e}^{2+}, V_{Z n}^{-}\right.$and $\left.V_{Z n}^{2-}\right)$. There is no experimental evidence of antisite defects in ZnSe (Laks et al., 1992) and hence these defects have not been considered in this analysis. This work takes into account the neutral, the singly and doubly charged $\mathrm{Zn}$ vacancies and interstitials. The neutral $\mathrm{Zn}$ vacancy has a threefold-degenerate level in the band gap and the possible charged states are $1-$ and $2-$, corresponding to the $\mathrm{Zn}$ vacancy levels acting as acceptor levels in undoped ZnSe. Similarly, the neutral Zn interstitial has two electrons occupying a single level in the band gap and the possible charge states are $1+$ and $2+$, and their levels act as donor states in the band gap of undoped ZnSe. The vacancies and interstitials of $\mathrm{Zn}$ having different charge states create different localized levels within the band gap and can also combine to form donor-acceptor pairs (DAP). Evidence of the existence of such DAPs has also been confirmed by photoluminescence (PL) measurements, discussed in greater detail in Section 3. 
A vapor-liquid-solid (VLS) growth mechanism accounts for the formation of ZnSe nanowires and their formation follows the reaction:

$$
Z n S e_{(s)}=Z n_{(v)}+\frac{1}{2} S e_{2(v)}
$$

The nanowire growth is driven by the chemical potential gradients between vapor, liquid and solid phase with growth taking place through a mass transfer across the liquid-solid interface. The system of nanowires is characterized by a temperature $\mathrm{T}$, at which there are a certain number of intrinsic point defects in the crystalline nanowires. The concentration of $\mathrm{Zn}$ vacancies and interstitials, $\left[V_{Z n}\right]$ and $\left[Z n_{i}\right]$, respectively, were estimated from (Laks et al., 1992; Reshchikov \& Morkoc, 2005):

$$
[V]=N_{\text {sites }} N_{\text {config }} \exp \left(\frac{S}{k}\right) \exp \left(-\frac{E_{f}}{k T}\right)
$$

where, $S$ is the entropy, $E_{f}$ is the formation energy of the defect, $N_{\text {sites }}$ is the number of sites in the lattice (per unit volume) where the defect can be incorporated (i.e., for $\mathrm{ZnSe}, N_{\text {sites }}=$ $\left.2.2 \times 10^{22} \mathrm{~cm}^{-3}\right), k$ is Boltzmann's constant, $\mathrm{T}$ is the absolute temperature and $N_{\text {config }}$ is the number of equivalent configurations in which the defect can be incorporated. The vacancy formation reactions and the corresponding mass-action relations are given in Table 1 . The concentrations of free electrons and holes are indicated by $p$ and $n$ and in reaction equations, free electrons and holes are indicated by $e^{-}$and $h^{o}$, respectively.

\begin{tabular}{|cccc|ccc|}
\hline $\begin{array}{l}\text { Reaction equations leading to } \\
\text { the formation of vacancies and } \\
\text { interstitials }\end{array}$ & \multicolumn{2}{|l|}{$\begin{array}{l}\text { Equations for equilibrium constants } \\
\text { based on mass-action relation }\end{array}$} \\
\hline $\begin{array}{c}Z n_{(v)}+ \\
\frac{1}{2}\left(S e_{2}\right)_{(v)}\end{array}$ & $=$ & $\mathrm{ZnSe}_{(s)}$ & $K_{f}$ & $=$ & $P_{Z n} P_{S e_{2}}^{1 / 2}$ \\
\hline$\frac{1}{2}\left(S e_{2}\right)_{(v)}$ & $=$ & $S e_{S e}^{x}+V_{Z n}^{2-}+$ & $K_{v}$ & $=$ & $\frac{\left[V_{Z n}^{2-}\right] p^{2}}{P_{S e_{2}}^{1 / 2}}$ \\
\hline$V_{Z n}^{2-}+h^{o}$ & $=$ & $V_{Z n}^{-}$ & $K_{Z n}^{\prime}$ & $=$ & $\frac{\left[V_{Z n}^{-}\right]}{\left[V_{Z n}^{2-}\right] p}$ \\
\hline$V_{Z n}^{-}+h^{o}$ & $=$ & $V_{Z n}^{x}$ & $K_{Z n}^{x}$ & $=$ & $\frac{\left[V_{Z n}^{x}\right]}{\left[V_{Z n}^{-}\right] p}$ \\
\hline$V_{Z n}^{2-}+Z n_{i}^{2+}$ & $=$ & 0 & $K_{f r}$ & $=$ & {$\left[V_{Z n}^{2-}\right]\left[Z n_{i}^{2+}\right]$} \\
\hline$Z n_{i}^{2+}+e^{-}$ & $=$ & $Z n_{i}^{+}$ & $K_{i}^{+}$ & $=$ & $\frac{\left[Z n_{i}^{+}\right]}{\left[Z n_{i}^{2+}\right] n}$ \\
\hline$Z n_{i}^{+}+e^{-}$ & $=$ & $Z n_{i}^{x}$ & $K_{i}^{x}$ & $=$ & $\frac{\left[Z n_{i}^{x}\right]}{\left[Z n_{i}^{+}\right] n}$ \\
\hline
\end{tabular}

Table 1. Equilibrium constants derived from reaction equations.

For the assumptions discussed above, the electrical neutrality condition is:

$$
n+2\left[V_{Z n}^{2-}\right]+\left[V_{Z n}^{-}\right]=p+2\left[Z_{i}^{2+}\right]+\left[Z n_{i}^{+}\right]
$$

The $\mathrm{Zn}$ partial pressure $\left(P_{\mathrm{Zn}}\right)$ and $S e_{2}$ partial pressure $\left(P_{S e_{2}}^{1 / 2}\right)$ in the vapor phase are governed by the equation:

$$
P_{\mathrm{Zn}} P_{\mathrm{Se}_{2}}^{1 / 2}=K_{f}
$$


where $K_{f}$ [in units of $(A t m)^{3 / 2}$ ] is the equilibrium constant for sublimation of ZnSe, which is a constant at a given temperature. During growth, the compound ZnSe source provides a minimum total pressure $P_{\min }$ over the growing nanowire in the growth chamber, ensuring that it remained an equilibrium $\mathrm{ZnSe}$ solid phase. The required minimum partial pressure of Se, $\left(P_{S_{2}}^{1 / 2}\right)_{\min }$ was calculated using the approximation:

$$
\left(P_{\mathrm{Se}_{2}}^{1 / 2}\right)_{\min } \approx\left(K_{f} / 2\right)^{1 / 3}
$$

To calculate the concentration of the six defects ([V $\left.V_{Z n}^{2-}\right],\left[V_{Z n}^{-}\right],\left[V_{Z n}^{x}\right],\left[Z n_{i}^{+}\right],\left[Z n_{i}^{2+}\right]$ and $\left.\left[Z n_{i}^{x}\right]\right)$, as a function of $\left(P_{S_{e_{2}}}^{1 / 2}\right)$, equations in Table 1 were modified and expressed in terms of the equilibrium constants. The evolution chart of the defects and the possible transitions with increasing Se vapor pressure $\left(P_{S_{2}}^{1 / 2}\right)$ is shown in Figure 1. The dependence of $\left[V_{\mathrm{Zn}}\right]$ and

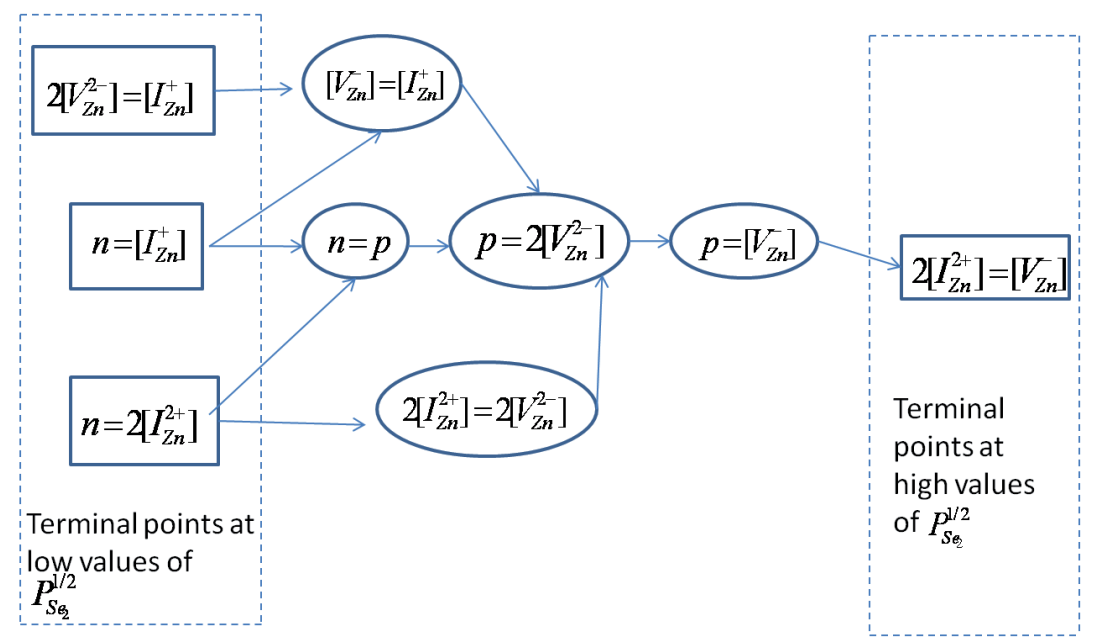

Fig. 1. Evolution chart of defects, where arrows refer to increasing $\left(P_{\mathrm{Se}_{2}}^{1 / 2}\right)$.

$\left[\mathrm{Zn} n_{i}\right]$ equilibrium concentrations on $\left(P_{\mathrm{Se}_{2}}^{1 / 2}\right)$ was established using the mass-action laws and charge neutrality conditions (Gool, 1966). Figure 2 shows the approximate solution of this set of equations. The approximation implies that in the ranges of $\left(P_{S_{e_{2}}}^{1 / 2}\right)$ pressure variation, three defect regimes $\left(2\left[Z n_{i}^{2+}\right]=2\left[V_{Z n}^{2-}\right], p=2\left[V_{Z n}^{2-}\right]\right.$, and $\left.p=\left[V_{Z n}^{-}\right]\right)$of the nine regimes shown in Figure 1 are considered. The concentration of $p,\left[V_{Z n}^{-}\right]$, and $\left[V_{Z n}^{x}\right]$ increases with $\left(P_{S_{2}}^{1 / 2}\right)$. As $\left(P_{S_{e_{2}}}^{1 / 2}\right)$ increases, $p$ increases and its concentration dominates causing a transition to $p=2\left[V_{\mathrm{Zn}}^{2-}\right]$ and finally at sufficiently high values of $\left(P_{S_{e_{2}}}^{1 / 2}\right), p=\left[V_{\mathrm{Zn}}^{-}\right]$will take over. The net concentration of defects as a function of $\left(P_{S_{e_{2}}}^{1 / 2}\right)$ is shown in Figure 3 for nanowires grown at $923 \mathrm{~K}$. It can be seen that nanowires grown under stoichiometric conditions, close to $\left(P_{\mathrm{Se}_{2}}^{1 / 2}\right)_{\min }$ have a net vacancy concentration of the order of $10^{17} \mathrm{~cm}^{-3}$. As the Se vapor pressure $\left(P_{\mathrm{Se}_{2}}^{1 / 2}\right)$ increases, it can be seen that the net vacancy concentration increases significantly. The predictions of the defect model (Figures 2 and 3) can be validated by structural, optical and opto-electronic characterization, described in greater detail in the following sections. 


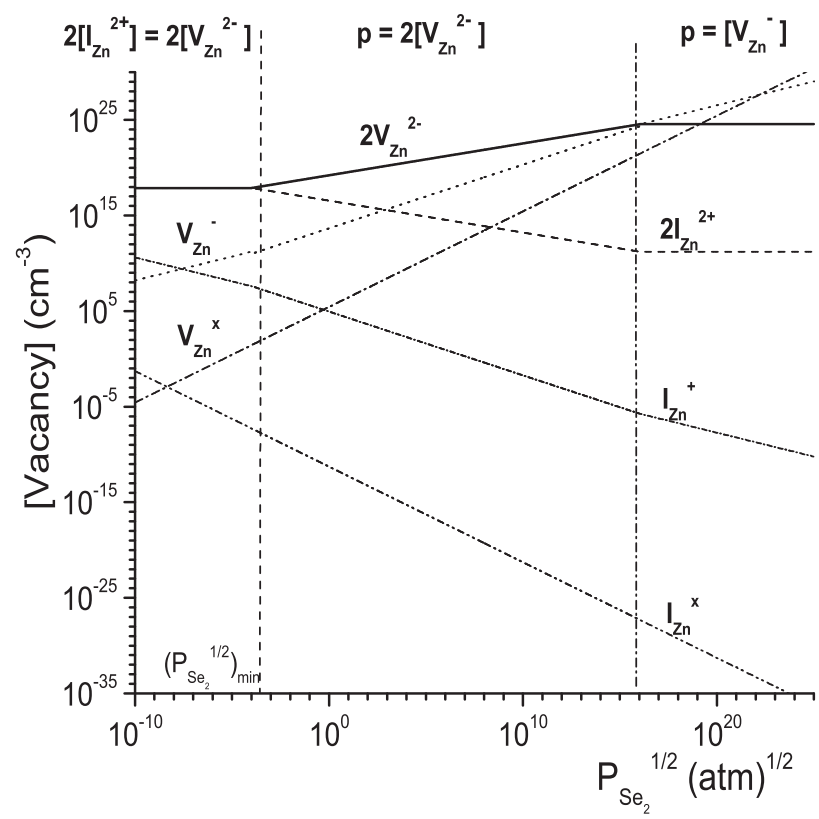

Fig. 2. Defect concentration as a function of Se vapour pressure $\left(P_{S_{2}}^{1 / 2}\right)$ for nanowires grown at $923 \mathrm{~K}$.

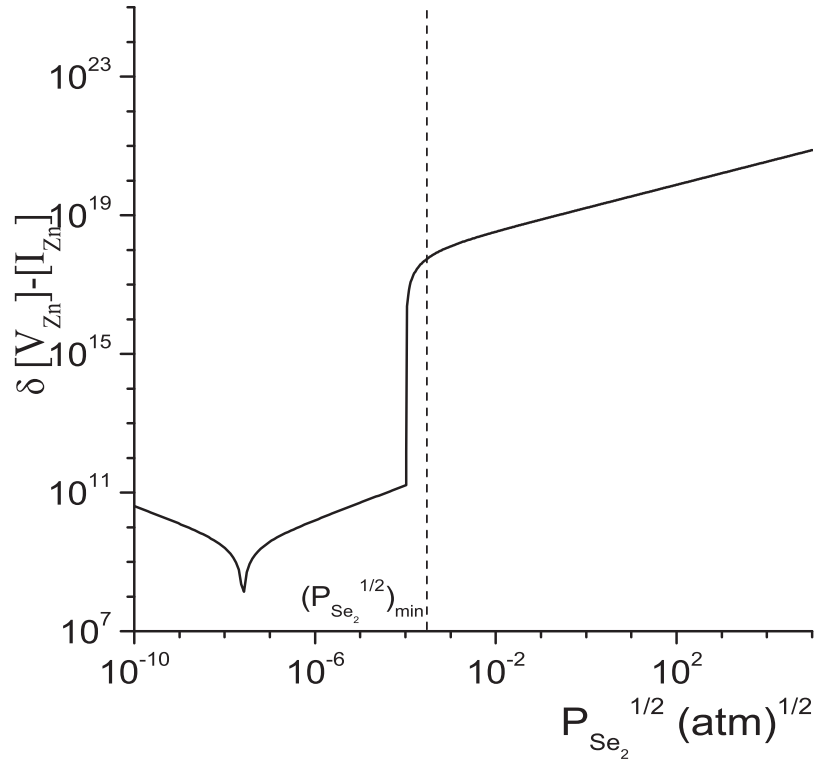

Fig. 3. Net defect concentration as a function of Se vapour pressure $\left(P_{\mathrm{Se}_{2}}^{1 / 2}\right)$ for nanowires grown at $923 \mathrm{~K}$. 


\section{ZnSe nanowire characterization}

Nanowire morphology and properties are dependent on growth parameters such as temperature, pressure as well as stoichiometry of the component fluxes. A non-stoichiometric component flux enhances the generation of intrinsic point defects that in turn affects the structural, electronic and optical properties of the nanowires. In the case of $\mathrm{ZnSe}$, an increase in the Se vapour flux was found to produce a high density of twinning defects. A brief review of structural, optical and opto-electronic properties of ZnSe nanowires and the dependence of these on growth conditions is presented.

\subsection{Structural characterization using electron microscopy}

Microstructural analysis of ZnSe nanowires grown under optimized conditions (Philipose, P.Sun, Xu, Ruda, Yang \& Kavanagh, 2007)show them to be structurally uniform and free from defects (Figure 4), although a few of the wires (especially those growing in the vicinity of the substrate edge) have planar defects.

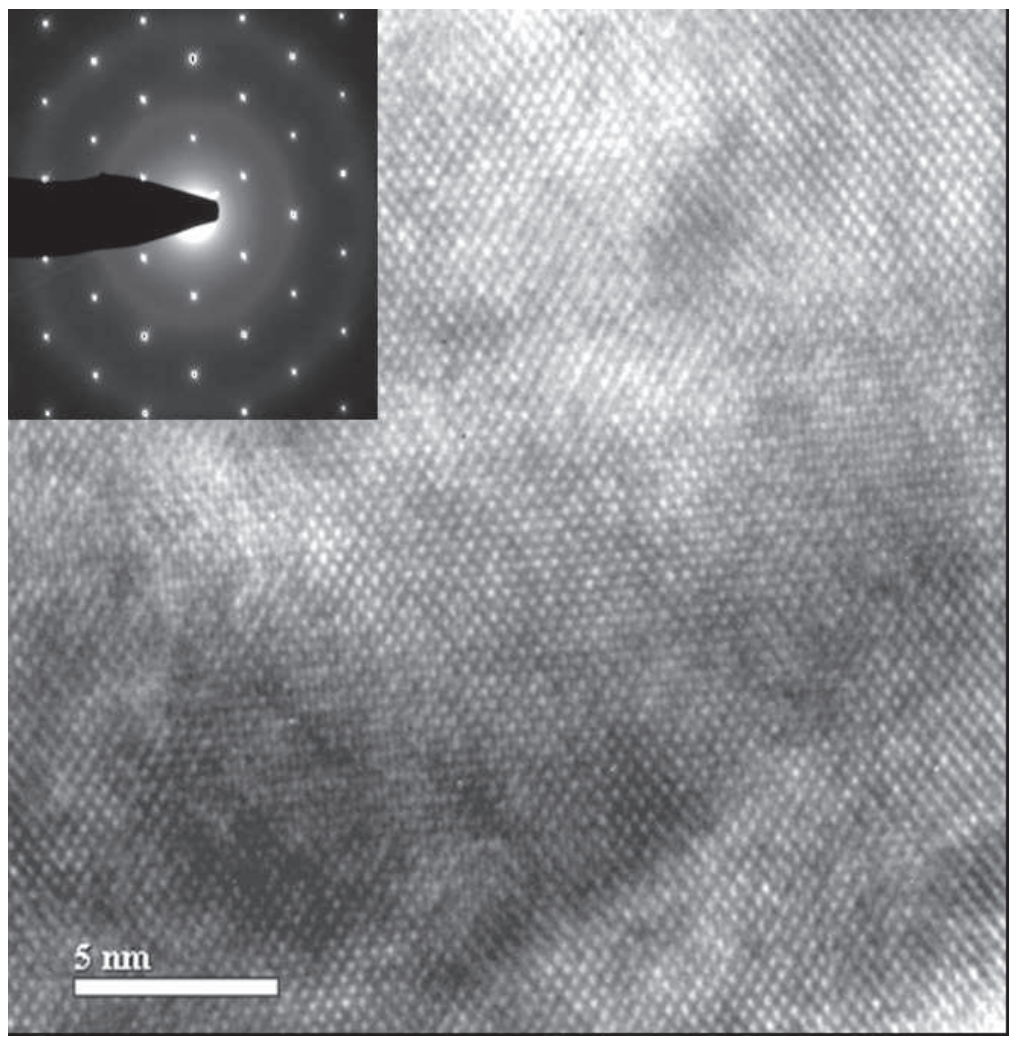

Fig. 4. Net defect concentration as a function of Se vapour pressure $\left(P_{S e_{2}}^{1 / 2}\right)$ for nanowires grown at $923 \mathrm{~K}$.

The growth direction for these nanowires is [111]. The planar defects are twins with $\{111\}$ twin planes, shown in Figure 5. Based on an analysis of numerous nanowires, it appears 

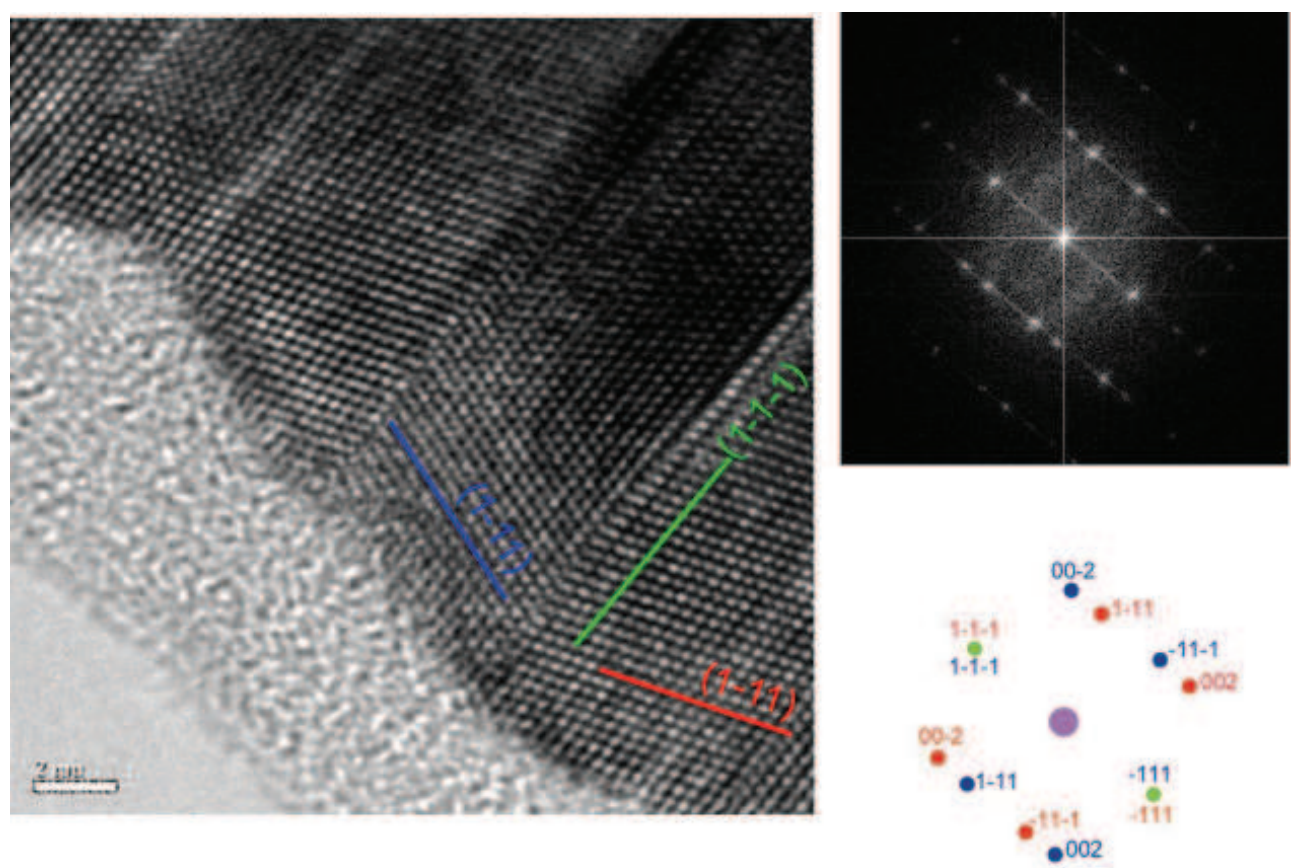

Fig. 5. HRTEM of non-periodic twins in a nanowire grown under Se rich conditions. SAED pattern and its Fourier transform are shown to the right. The drawing, with different colour spots, is indexed. The colour corresponds to colour of line in the HRTEM image.

that fluctuations in growth temperature, size and shape of Au catalytic droplet influence the formation of these defects. Thermal stress during the growth process, residual oxygen and vapor phase non-stoichiometry above the growing nanowires can all contribute to twinning. The high ionicity of ZnSe has also been discussed as a factor contributing to the propensity for this material to twin. In order to understand the dependence of growth direction on temperature, the nanowire has been considered as a column with side surfaces. The total energy of a nanowire includes contribution from the surfaces and from the bulk. Due to the large surface to volume ratio of the nanowires, an important factor controlling the growth orientation is the surface energy. In reference (Chan et al., 2006), the surface energies of the major facets of ZnSe crystal were estimated and though the values for different surfaces were almost comparable, the trend appears to be: $\gamma_{111 B}<\gamma_{100}<\gamma_{311}<\gamma_{110}$. At low growth temperatures, the area of the side surfaces is relatively insignificant, and hence the contribution from the side surfaces can be ignored. In this case, the nanowires will prefer to grow along the [111] direction, due to the fact that the (111) interface has the smallest surface energy. At higher temperatures, the relative contribution from the side surfaces will be significant and preference is given by the system to the growth direction that will lower the total system energy. The relative importance of surface energy, substrate orientation and role of Au-alloy tip in controlling the growth process remains an open question. For compound semiconductors, the surface energy is not uniquely defined, but rather depends on the relative concentration of constituents through their chemical potentials. The presence of more than 
one morphologically distinct nanostructure for a given growth condition suggests that several factors can be contributing to the growth process, with relatively small energy differences involved.

\subsection{Optical characterization using photoluminescence spectroscopy}

A typical room temperature photoluminescence (PL) spectrum of ZnSe nanowires has two characteristic emission peaks - a band edge (BE) emission peak at $2.68 \mathrm{eV}(463 \mathrm{~nm})$ and a broad deep defect (DD) emission peak in the region $1.8-2.4 \mathrm{eV}(500 \mathrm{~nm}$ to $680 \mathrm{~nm})$. The origin of the DD emission peak has been attributed to several recombination mechanisms: (i) donor-acceptor pairs (DAPs) involving Zn vacancies and Zn interstitials Philipose, Yang, Xu \& Ruda (2007); (ii) DAP transitions caused by thermal exciton dissociation due to laser induced zinc vacancy (Martinez-Canton et al., 2005), etc.. In this section, we review the findings by Philipose et al., (Philipose, Yang, Xu \& Ruda, 2007) in identifying the origin of the red luminescence band in ZnSe nanowires. To facilitate this study, the PL spectra of nanowires grown under three different growth conditions were obtained and is shown in Figure 6. The emission spectrum of nanowires grown under near stoichiometric conditions, when the Se vapor pressure is close to $\left(P_{\mathrm{Se}_{2}}^{1 / 2}\right)_{\min }$ (curve 2 in Figure 6) shows that the spectrum is comprised of two emission bands with a narrow band edge (BE) peak at $463 \mathrm{~nm}$ and a DD band extending from $\sim 500 \mathrm{~nm}$ to $680 \mathrm{~nm}$. The BE emission peak intensity in some cases is stronger than the DD emission intensity centered at $\sim 620 \mathrm{~nm}$. As the Se vapor pressure is increased $\left(\left(P_{S_{2}}^{1 / 2}\right)\right.$ is increased), the emission spectra (curve 3 in Figure 6) shows a complete disappearance of the BE emission peak and the PL spectrum is dominated by the DD emission peak. The amplitude of the BE emission is insignificant. These findings are consistent with the defect model predictions shown in Figure 3, that as $\left(P_{\mathrm{Se}_{2}}^{1 / 2}\right)$ increases, the net vacancy concentration increases. Another interesting feature of the plot shown in Figure 3 is that as the partial pressure of Se vapor is reduced from $\left(P_{\mathrm{Se}_{2}}^{1 / 2}\right)_{\min }$, there is a significant decrease in the net concentration of defects by several orders of magnitude. This was verified experimentally by growing ZnSe nanowires under Zn-rich conditions, i.e., when the partial pressure of Se is lower than $\left(P_{S_{2}}^{1 / 2}\right)_{\text {min }}$. The PL spectra (curve 1 in Figure 6) shows an enhanced BE emission with virtually no emission from the defect states. In comparison with curve 2 in Figure 6, there is an enhancement in the ratio of $\mathrm{BE} / \mathrm{DD}$ emission by more than three orders of magnitude, in agreement with the predictions of the defect model.

\subsection{Origin of the deep defect emission band in PL spectra}

In PL spectroscopy, the simplest recombination process is a band-to-band recombination where a free electron from the conduction band recombines radiatively with a free hole from the valence band. Impurities or defects which introduce traps, donor or acceptor levels in the band gap provide alternate paths for recombination. When both the excited electron and hole are captured by different impurity centers and then the trapped electron and hole recombine radiatively, the process is a 'donor-acceptor' pair (DAP) recombination. Emission bands thus appear in the low energy region of the spectrum resulting from recombination of electron and hole pairs captured at acceptor and donor sites respectively. Recombination of this type reflects the energy difference between the donor and acceptor levels involved in the recombination process involving distant DAPs. The energy of the emission peak 
corresponding to recombination of DAPs is given by the equation:

$$
\hbar \omega_{m}=E_{g}-\left(E_{d}+E_{a}-\frac{e^{2}}{4 \pi \epsilon \epsilon_{o} R_{m}}\right)
$$

where, $\epsilon$ is the permittivity of $\mathrm{ZnSe}=8.8\left(\epsilon_{0}\right), \epsilon_{0}$ is the permittivity of free space $=8.85$ $\times 10^{-14} \mathrm{~F} / \mathrm{cm}, R_{m}$ is the distance between partners of the DAP and this determines the Coulomb interaction energy (the last term in equation 6), $E_{g}$ is the energy band gap of $\mathrm{ZnSe}$ $=2.7 \mathrm{eV}$ at room temperature; and $E_{d}$ and $E_{a}$ are the activation energies of the donors and acceptors. From equation 6, it is also evident that for small separation between donor and acceptor atoms, the energy of the DAP peak is substantially higher than that of the distant pairs. Hence, with DAP recombination, the actual spectral emission curve will depend on the opposing influence of pair distribution function and pair recombination transition probability - there are likely a large number of distant pairs, but their recombination probability will be low, while there will be few closely separated pair which will have a high recombination probability. In this case, the shape of the PL spectrum results from the opposing influence of

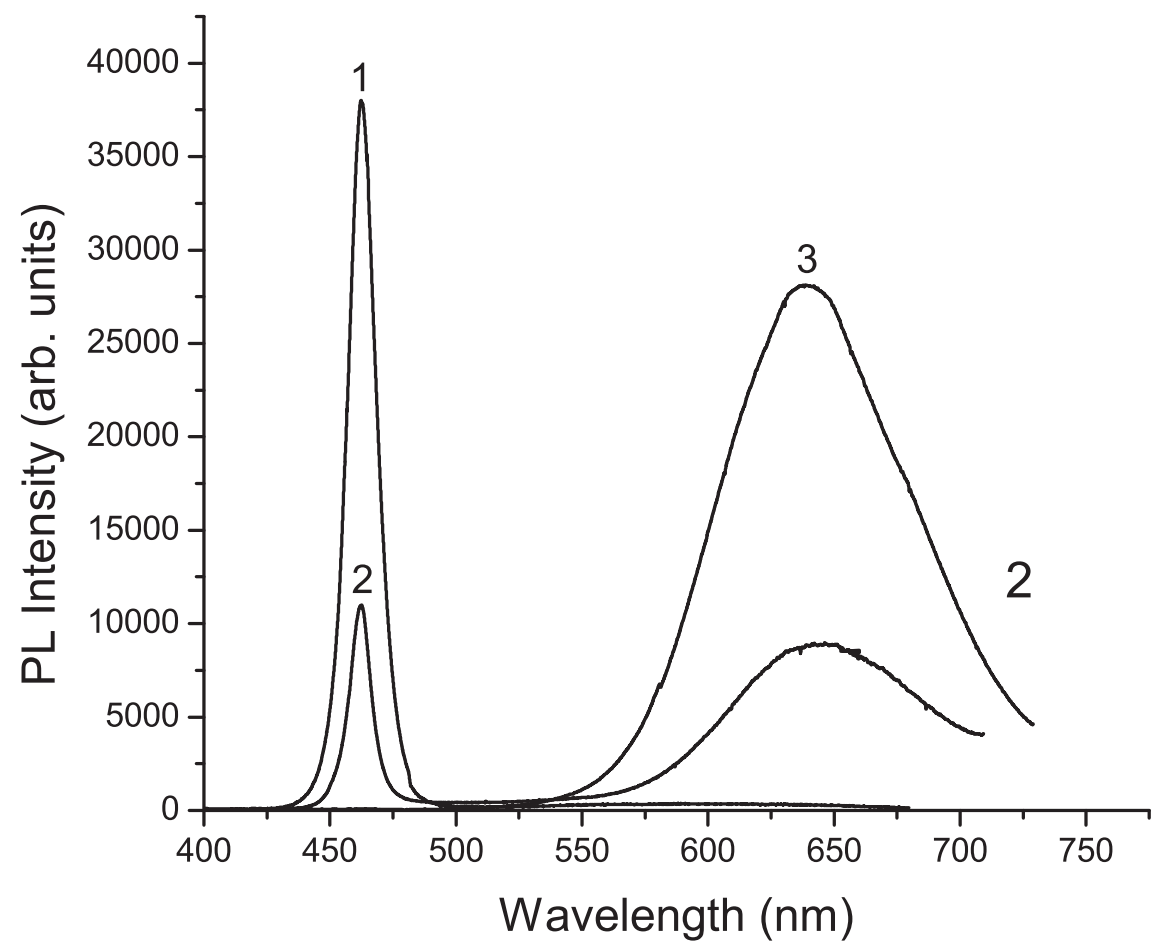

Fig. 6. Room temperature PL spectra of ZnSe nanowires grown under different growth conditions; Curve 1: When Se vapour pressure is less than $\left(P_{S_{2}}^{1 / 2}\right)_{\min }$, the spectrum is dominated by BE emission; Curve 2: When Se vapour pressure is close to $\left(P_{S_{e_{2}}}^{1 / 2}\right)_{\min }$, there is a significant decrease in the intensity of the $\mathrm{BE}$ emission and there is comparable DD emission; Curve 3: When the Se vapour pressure is greater than $\left(P_{S_{2}}^{1 / 2}\right)_{m i n}$, the PL spectrum shows strong DD emission and no emission from the BE states. 
the DAP distribution function and the pair recombination transition probability. Studies on bulk ZnSe attribute the origin of the DD emission band (red luminescence band) to such DAP

transitions. Vacancies and interstitials having different charged states $\left(V_{S e}^{+}, V_{S e}^{2+}, V_{\mathrm{Zn}}^{-}, V_{\mathrm{Zn}}^{2-}\right.$, $Z n_{i}$ and $S e_{i}$ ) will thus introduce different localized energy levels within the band gap and also combine into donor-acceptor pairs (DAPs). The origin and mechanism of formation of the DD emission band in ZnSe nanowires is further explored to provide information on type, energy and concentration of the defects that contribute to it.

The broad defect band shown in Figure 6 is most likely formed by many closely spaced emission lines due to recombination of DAPs with large separation between them and the shape results from the opposing influence of the pair distribution function and the pair recombination transition probability. This emission band was for the first time treated quantitatively (for bulk ZnSe) in Reference (Zacks \& Halperin, 1972) using experimental evidence and an expression was derived for the dependence of the peak energy of the DD band on excitation intensity. On increasing the excitation intensity, the peak of the broad $\mathrm{DD}$ emission band (corresponding to DAP recombination) shifts to higher energies. In the following section, a detailed study of the dependence of excitation intensity on DD emission for $\mathrm{ZnSe}$ nanowires is presented.

\subsubsection{Dependence of peak position of DD emission band on intensity of laser excitation}

To study this dependence, the excitation intensity was varied from about $0.07 \mathrm{~mW}$ to more than $2.5 \mathrm{~mW}$ for nanowires grown under high Se vapor pressure and for those grown under near stoichiometric conditions, and the shift of the peak position of the DD emission band was studied. Figure 7 shows the influence of excitation intensity, $I_{e x}$, on the DAP peak energy, $h v_{m}$. As can be seen in the figure, there is a shift in the DAP peak energy to higher energy values with increasing excitation intensity. According to Zacks and Halperin (Zacks \& Halperin, 1972), the peak energy of the DAP emission $\left(h v_{m}\right)$ is related to the excitation intensity J by the following equation:

$$
I_{e x}=D\left[\frac{\left(h v_{m}-h v_{i}\right)^{3}}{\left(h v_{B}+h v_{i}-2 h v_{m}\right)}\right] \times \exp \left[-2 \frac{\left(h v_{B}-h v_{i}\right)}{\left(h v_{m}-h v_{i}\right)}\right]
$$

where $I_{e x}$ is the excitation intensity, $h v_{m}$ is the peak energy of the DAP transition spectrum, and $h v_{i}$ is the emission energy for infinite R. $h v_{i}$ is defined by the equation:

$$
h v_{i}=E_{g}-\left(E_{A}+E_{D}\right)
$$

The term $h v_{B}$ is defined by the equation

$$
h v_{B}=E_{B}+h v_{i}
$$

where

$$
E_{B}=e^{2} / 4 \pi \epsilon \epsilon_{o} r_{B}
$$

where, $E_{B}$ is the binding energy of the DAP, $r_{B}$ is the shallow impurity Bohr radius, and $\mathrm{D}$ is a constant. In the low excitation limit, the DAP peak energy approaches the value of $h v_{i}$. The values obtained experimentally (Figure 7) were used for calculating the parameters appearing in equation 7 . Using the best-fit line, the value of $E_{B}=18 \pm 0.5 \mathrm{meV}$ is obtained for nanowires grown under high Se vapor pressure.

From equation 10, the value of $r_{B}$ is estimated to be $9.1 \pm 0.2 \mathrm{~nm}$ for the shallow impurity Bohr radius in nanowires grown under high Se vapor pressure. The value of $E_{i}$ is around $1.95 \mathrm{eV}$, 
giving $E_{d}$ of $\sim 0.35 \mathrm{eV}$ and $E_{a}$ of $\sim 0.40 \mathrm{eV}$. Using the value of $r_{B}$, the concentration of the minority defect species, [V], can be calculated by using the equation:

$$
[V]=\frac{3}{4 \pi r_{B}^{3}}
$$

Hence, the concentration of defects under high Se vapor pressure is $3.1 \times 10^{17} \mathrm{~cm}^{-3}$, in excellent agreement with the defect analysis predictions described in Section 2.

The characteristic shift of the DAP peak energy with excitation intensity follows from the fact that at high excitation intensities, widely separated DAPs will be saturated because of their lower transition probability and hence a large fraction of DAPs with smaller radius will be excited. These will then decay radiatively due to their higher transition probability. Hence, an increase in the excitation intensity results in an increase in the relative intensity of PL emission from DAPs with smaller Bohr radius, leading to the observed shift in the DAP peak energy to higher energies as the excitation intensity increases.

Using the above analysis, as well as results obtained from PL measurements, the strong red $\mathrm{DD}$ emission in $\mathrm{ZnSe}$ is identified as due to a DAP recombination mechanism involving $V_{Z n}$ as the acceptor species and $V_{S e}$ or $Z n_{i}$ as the donor species. In order to identify the second species in the DAP contributing to the broad DD emission at $1.95 \mathrm{eV}$ and to confirm the presence of Zn vacancies, the as-grown nanowires were subjected to post-growth treatment.

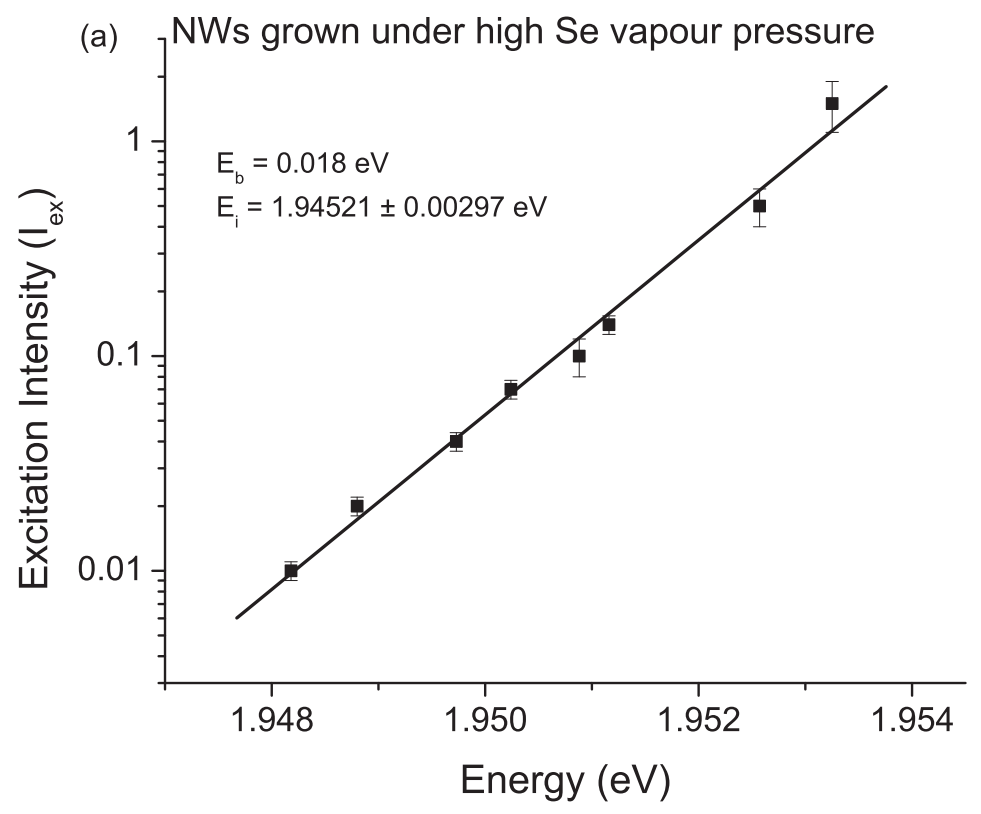

Fig. 7. Dependence of peak energy of the DAP recombination emission band on excitation intensity for growth under high Se vapour pressure. 


\subsubsection{Post-growth treatment of $\mathrm{ZnSe}$ nanowires to confirm the role of $\mathrm{Zn}$ vacancies in DD emission}

In order to confirm the role of $V_{Z n}$ in the DD emission, as-grown $\mathrm{ZnSe}$ nanowires were annealed at $650^{\circ} \mathrm{C}$ in a $\mathrm{Zn}$-rich atmosphere under a constant flow of Ar gas. Figure 8 shows a comparison of the PL spectra for samples before and after Zn-vapor treatment for 30 minutes and 45 minutes, respectively. A considerable enhancement in the ratio of the $\mathrm{BE}$

(a)

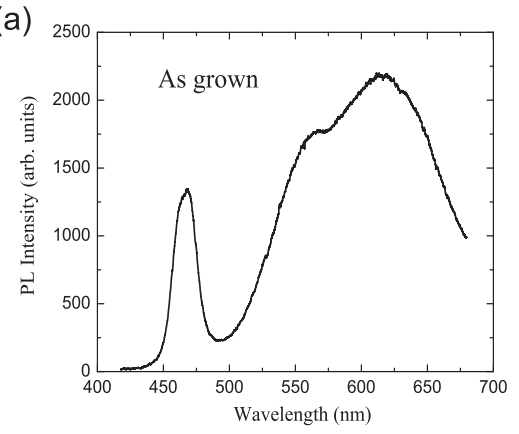

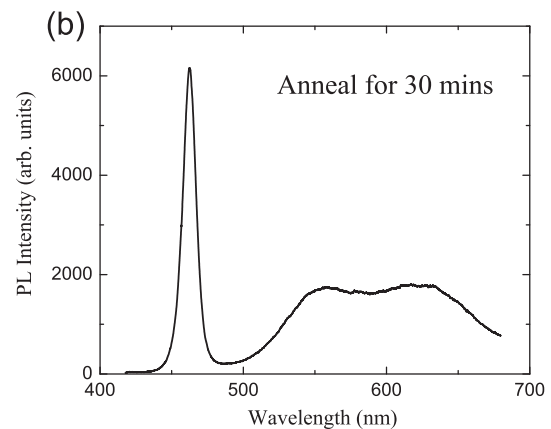

(c)

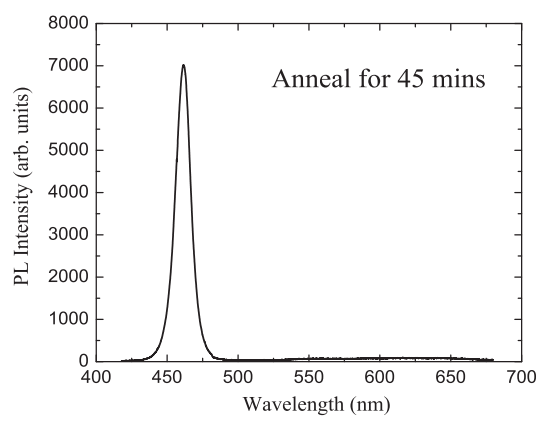

Fig. 8. PL spectra of ZnSe nanowires at room temperature: (a) as-grown nanowires; (b) after treatment in a $\mathrm{Zn}$-rich atmosphere at $650^{\circ} \mathrm{C}$ for 30 minutes, and (c) after treatment in a Zn-rich atmosphere at $650^{\circ} \mathrm{C}$ for 45 minutes.

to DD emission intensity can clearly be seen as a result of the annealing process. This ratio increases with annealing time from $\sim 0.6$ to 87 times. This enhancement may be explained by a reduction in the concentration of $V_{Z n}$ owing to annealing in a Zn-rich atmosphere, and hence a reduction in the concentration of possible pairs contributing to DAP emission. The temporal dependence thus corresponds to the diffusion of $\mathrm{Zn}$ from the vapor into the nanowires. This process has a strong dependence on temperature as is shown in Figure 9. This temporal dependence of BE/DD intensity shows that $\mathrm{Zn}$ atoms migrate or 'self-diffuse' through the ZnSe crystal lattice by a mechanism involving vacancies or interstitials. Analyzing the temperature dependence of $\mathrm{Zn}$ diffusion from the vapor into the ZnSe nanowires (Figure 9), the activation energy is estimated to be $0.6 \mathrm{eV}$. Earlier reports on zinc diffusion in $\mathrm{ZnSe}$ (Rong et al., 1996) estimates the activation energy for interstitial diffusion to be $\sim 0.6-0.8 \mathrm{eV}$ and that 


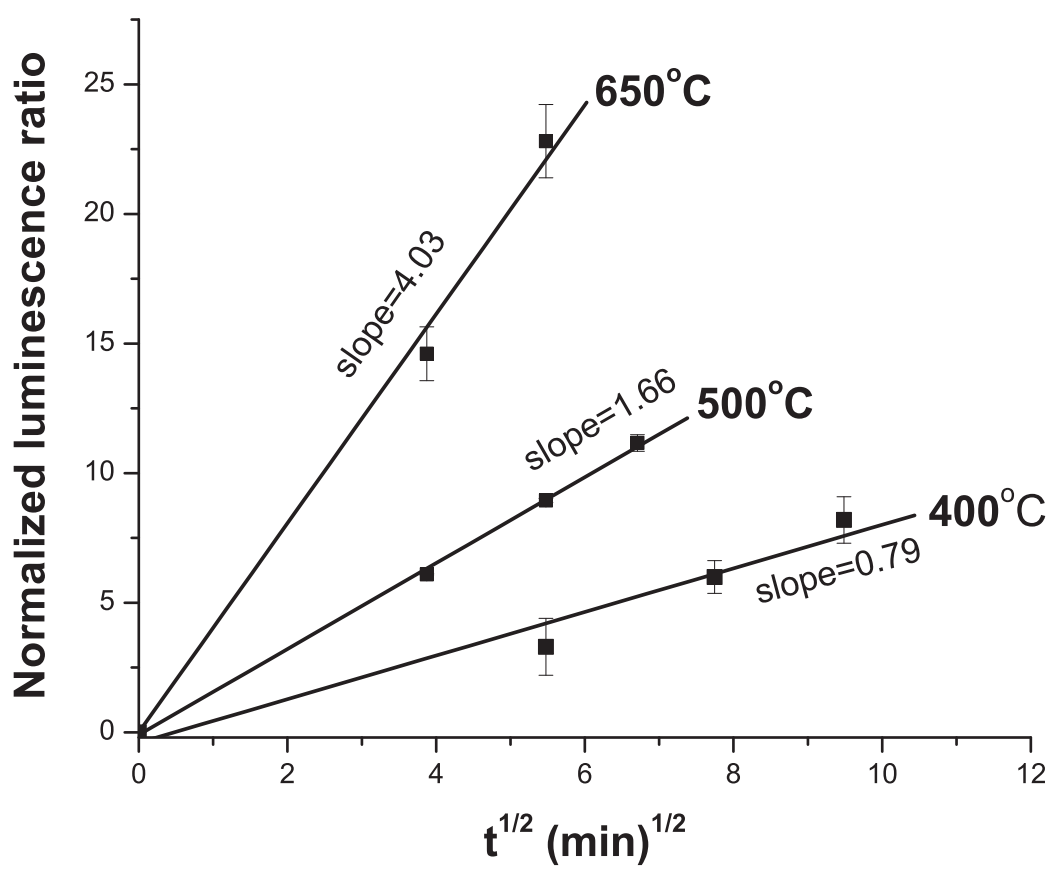

Fig. 9. Effect of temperature on ratio of BE to DD emission intensity of ZnSe nanowires. Nanowire diameters are in the range $80-90 \mathrm{~nm}$.

for vacancy diffusion to be $\sim 1.26 \mathrm{eV}$ (Watkins, 1976). The activation energy of $0.6 \mathrm{eV}$ is hence considered suggestive of the fact that zinc interstitials are the mobile species in the diffusion mechanism. As the nanowires are annealed in $\mathrm{Zn}$ atmosphere, $\mathrm{Zn}$ interstitials diffuse into the vacancy sites and effectively reduces the concentration of DAPs responsible for the DD emission band.

\subsubsection{Carrier dynamics in ZnSe nanowires}

Studies on the ultrafast carrier dynamics can provide important insights into the role played by intrinsic point defects in the optical properties of nanowires. In Reference (Othonos et al., 2007), the authors present their results on transient absorption measurements for stoichiometric and defect-rich ZnSe nanowires, following ultrafast excitation above the band gap. Results of transient absorption measurements on ZnSe nanowires, excited at $400 \mathrm{~nm}$ is shown in Figure 10. When the nanowires were probed at wavelengths below the band gap, there was a sharp decrease in the absorption following the excitation pulse. This was followed by an exponential recovery within a few picoseconds. The opposite behavior is observed for probing wavelengths above the band gap. In this case, there is a sharp increase in absorption followed by a recovery to equilibrium. When probing at $450 \mathrm{~nm}$, which is very close to the ZnSe band edge, the induced absorption has a very long recovery to its equilibrium value. This observation is explained on the basis that when a semiconductor is excited with photons 


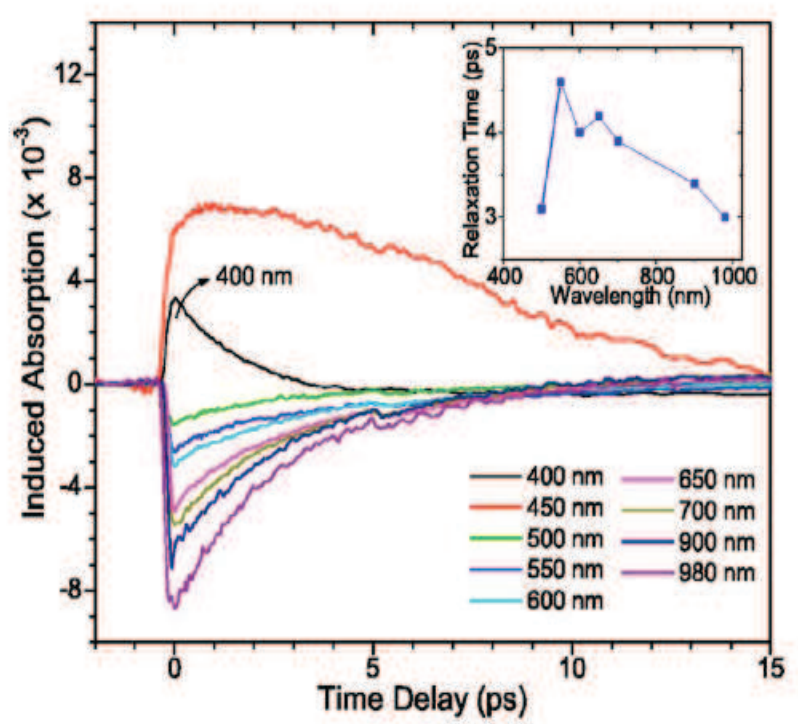

Fig. 10. Time-resolved induced absorption for near stoichiometric ZnSe nanowires. The sample is excited at $400 \mathrm{~nm}$ and probed using white light continuum generation. Inset shows the various relaxation rates for the different probing wavelengths.

of energy greater than its band gap, carriers generated within the conduction and valence band will relax to lower energy states within the bands or energy states below the bands originating from IPDs. These photogenerated carriers will contribute towards two competing effects; state filling (SF) and photoinduced absorption (PA). In the SF effect, the occupancy of energy states by the photogenerated carriers will result in a time dependent reduced linear absorption and thus enhanced transmission. In the PA effect, the probing beam will cause carriers occupying certain energy states to undergo secondary excitation to higher energy states resulting in an increase in linear absorption. The amplitude of this increase will depend on the coupling efficiency between the energy states and the number of carriers present in the lower coupled energy states. SF and PA are competing effects and can occur at the same time, thus affecting the intensity of the absorption signal, as is seen in Figure 10. In the stoichiometric ZnSe nanowires under discussion, the large concentration of defects inherent in this material accounts for the fact that SF effect is not observed when probed in the range from $450-500 \mathrm{~nm}$. In this range, there is an increase in the temporal absorption due to secondary excitation of the photo-generated carriers. For probing wavelengths in the range of $500-550$ $\mathrm{nm}$, the transient absorption changes are very small, which could be due to the absence of energy states within this probed region or due to the competing effects of state filling and secondary excitation. When probing above $550 \mathrm{~nm}$ (below the band gap), the SF effects dominate and is attributed to the occupation of the IPD energy states causing an observed reduction in the absorption. The observed negative induced absorption will eventually return 
to its equilibrium value as the photogenerated carriers move out of these energy states. The relaxation rate for these carriers out of the IPD states is estimated to be $\approx 3-4$ ps.

\section{Electron transport mechanism in nanowires}

Transport characteristics refer to the motion of electrons in response to an electric field. The two basic processes of current conduction are diffusion of carriers due to a gradient in carrier concentration, and drift of carriers due to the electric field. In 1D electron systems, drift and diffusion of electrons are equally important and any charge distribution relaxes according to the diffusion law with some effective diffusion coefficient enhanced due to the drift phenomena. During transport, the electrons always suffer scattering by impurities, crystal imperfections or with the lattice. In a nanowire, as the diameter of the wire decreases, electrons in the wire become increasingly confined. This affects the scattering of the electrons and causes a change in the electron transport mechanism. Electron transport can occur in the ballistic, diffusive or localized regimes, depending on the system size. In the localized regime, the system is highly disordered and the wave function becomes localized, i.e. the particle gets trapped and transport occurs by hopping. In the ballistic regime of transport, electrons can propagate through the material without scattering and current is determined by the ballistic motion of carriers in the electric field. In the diffusive regime, electrons suffer many scattering events. As the length scale (L) of the wire is reduced to the mean free path $(l)$ of the electrons, the electron transport mechanism changes from diffusive to ballistic. Electrical conductivity of undoped nanowires is to a large extent controlled by the presence of impurity atoms and defects in the semiconductor crystal lattice. The localized electronic states related to these defects can limit the characteristics of the material by acting as scattering or recombination centers for the charge carriers.

\subsection{Transport properties of ZnSe nanowire array}

ZnSe nanowire arrays for this study were fabricated by Au-catalyzed VLS mechanism. The nanowires in the array had diameters in the range of $80-150 \mathrm{~nm}$ and lengths of several micrometers. The estimated wire density in the array is $\approx 10^{6}$ wires $/ \mathrm{mm}^{2}$. The voids between the wires were filled with 2\% PMMA (poly-methyl-methacrylate) in Anisole, which serves as a dielectric. This ensures that the array of nanowires is encapsulated in a dielectric matrix which isolates individual nanowires and forms an insulating layer between the top and bottom electrodes. Au electrodes $(1 \mathrm{~mm} \times 1 \mathrm{~mm})$ were evaporated on top of the encapsulated array of nanowires. The back contact to the nanowire array was established using the heavily doped Si substrate. Electrical characterization of the nanowire array was performed using an HP4140B meter at temperatures in the range of 90-400 K. The measured current-voltage (I-V) characteristics are shown in Figure 11. As can be seen, the I-V characteristics have a symmetric and essentially superlinear character with increasing bias. Superlinear I-V characteristics similar to those shown in Figure 11 have been observed in semiconductor nanowires by several groups. This non-linearity cannot be attributed to contact phenomena, for the samples discussed here, where the two contact materials are essentially different, while the I-V characteristic has a symmetric character. The top Au electrode is to the array of nanowires, while the bottom electrode is an indium contact established to the heavily doped substrate. The I-V characteristics are explained by wire non-uniformities (Philipose et al., 2006). In order to explain this observed phenomena, the conductivity of a single nanowire with carrier concentration varying randomly along the wire is considered: $n=n(z)$ and, for simplicity, with a coordinate-independent mobility $\mu$. For relatively low carrier concentration 


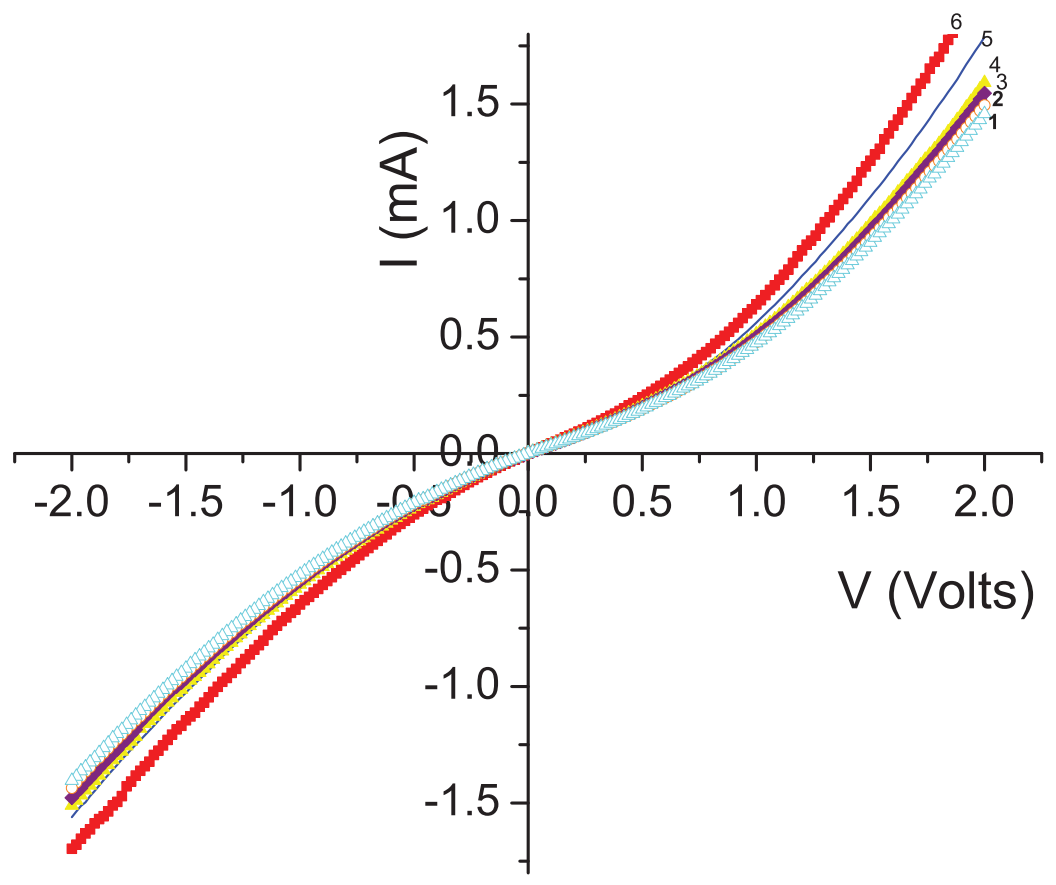

Fig. 11. Experimental current-voltage (I-V) characteristics at the temperatures $\mathrm{T}$ (in K): 90 (1); 140 (2); 200 (3); 250 (4); 350 (5); 400 (6)

samples, this is a reasonable assumption, based on the dependence of $\mu$ on $\mathrm{n}$. For a small applied voltage $\mathrm{V}$, we may consider a wire as a series connection of segments with different conductivities, so that its total resistance is given by:

$$
R_{o}=\frac{1}{e \mu} \int \frac{d z}{n(z)}
$$

For the case of very large fluctuations in $n, R_{o}$ will be determined by the region with minimal $n$, or the so-called 'weakest link'. High electric fields applied to the nanowire array will distribute carriers in the sample more uniformly, and, if the contacts are not injecting, such that the total number of carriers does not change, then when $V \rightarrow \infty$, the distribution becomes uniform and the resistance tends to a limiting value:

$$
R_{1}=\frac{L^{2}}{e \mu}\left[\int n(z) d z\right]^{-1}
$$

where $L$ is the wire length. For arbitrary $n(z), R_{o}$ is always greater than $R_{1}$ and the I-V characteristic has a superlinear character (Philipose et al., 2006). The above discussion is for a single nanowire, and the array comprises of many such nanowires contacted by two electrodes 
and hence represents a parallel connection of many nanowires and what is measured in Figure 11 is the average conductivity of the nanowires. Details of the inhomogeneity in conductivity of the nanowires comprising the array and its temperature dependence is presented in Reference (Philipose et al., 2006).

A very important property distinguishing this model from other models, also giving a superlinear I-V characteristic (e.g., contact barrier), is the behaviour of differential conductivity $d I / d V$, which, being an increasing function of $V$ at both $V \rightarrow 0$ and $V \rightarrow \infty$, tends to constants of $1 / R_{o}$ and $1 / R_{1}$ respectively. The value of the differential conductance, determined from the measured I-V characteristics (Figure 12) shows the values of $d I / d V$ obtained from the positive branches of the experimental curves of Figure 11 after smoothing. All the curves demonstrate a distinctive trend to saturation at $V \rightarrow 0$ and $V \rightarrow \infty$, which confirms the hypothesis of inhomogeneity-related non-linearity of the I-V characteristic.

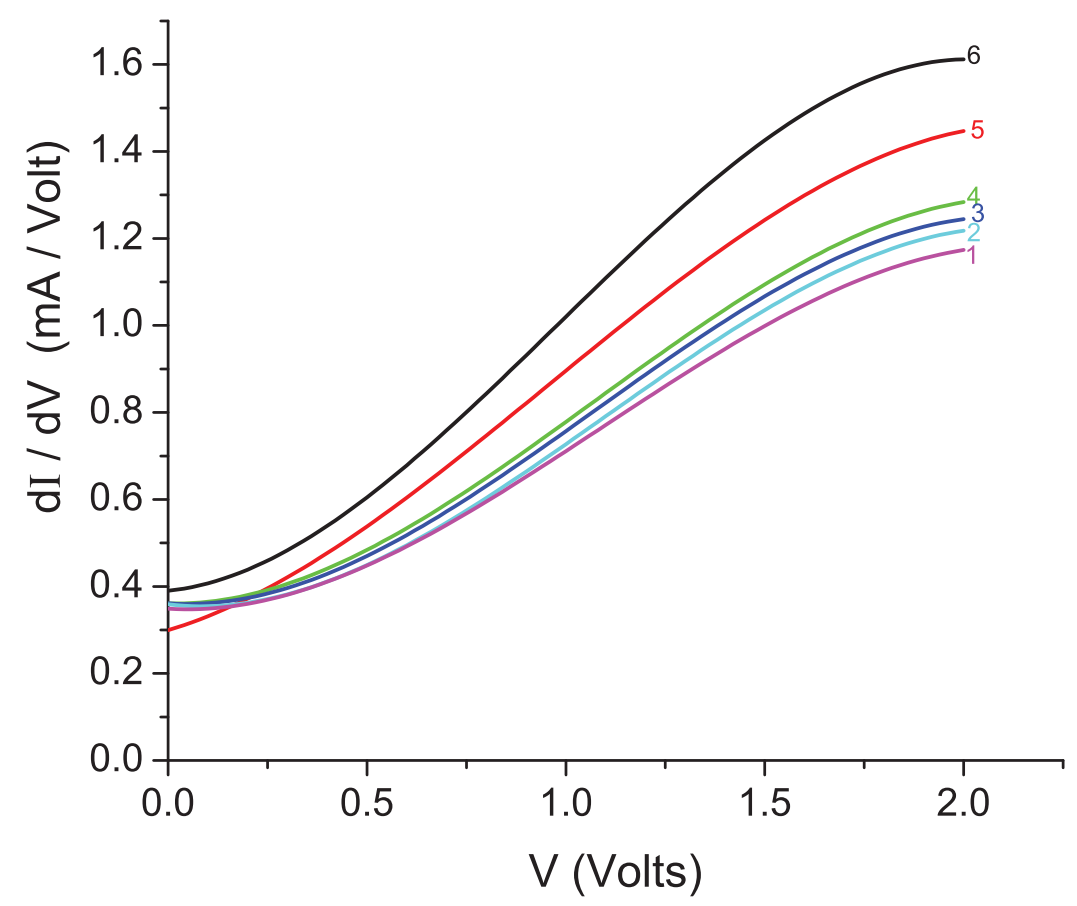

Fig. 12. Differential conductance $(d I / d V)$ for positive bias.

\subsection{Photoconductivity measurements on ZnSe nanowire array}

Photocurrent measurements were performed on the nanowire array using a chopped halogen lamp source and scanning monochromator that covers the spectral range from 400 to $550 \mathrm{~nm}$, with a lock-in amplifier. The spectral response of the ZnSe nanowire array (Figure 13) shows a threshold wavelength at about $463 \mathrm{~nm}$, corresponding well to band edge emission for bulk $\mathrm{ZnSe}$. The array under study was comprised of wires with diameters in the range of 80-150 $\mathrm{nm}$, and due to their large diameters they should not be noticeably influenced by the size 


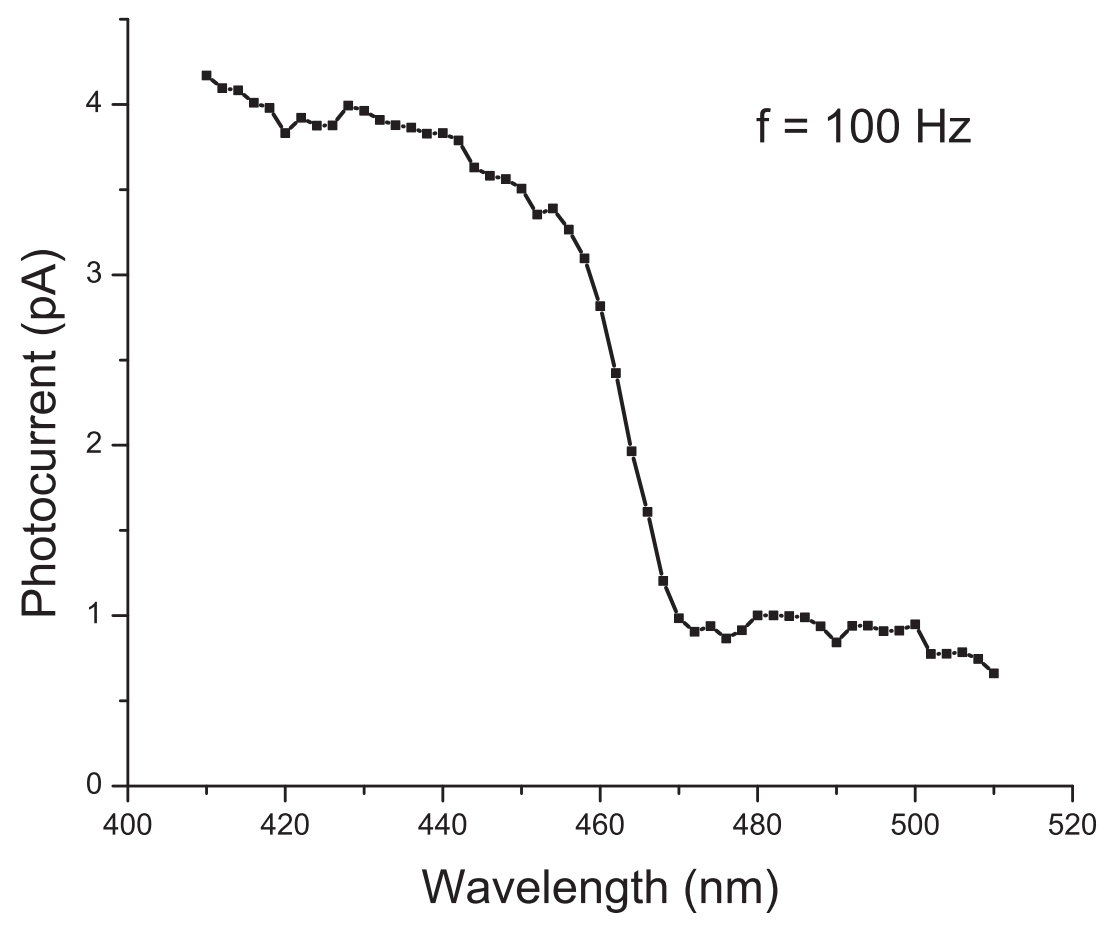

Fig. 13. Photocurrent as a function of wavelength for the ZnSe nanowire array.

quantization. The photoresponse of the ZnSe nanowire array also confirms that the nanowires are well contacted by the top and the bottom electrodes, as a photocurrent in proportion to the illumination flux is measured for these low excitation conditions. The frequency dependence of the photoresponse (Figure 14) shows that the amplitude of the photocurrent reduces with increasing modulation frequency. This dependence may be attributed to a process with characteristic time $\tau=3 \mathrm{~ms}$, presumably related to deep centre capture.

\section{Surface damage caused to nanowires subjected to laser irradiation}

In the optical characterization techniques discussed above, the nanowires were excited using focused laser light at room temperature and in air. It was observed that after prolonged exposure to the laser irradiation, there was a quenching of the NBE emission in the nanowires as shown in Figure 15. Possible factors that could be responsible for this phenomenon are sample heating by laser beam leading to photo-oxidation of the nanowires or radiation enhanced processes including laser-induced point defects generated at the surface of the nanowires. The large surface to volume ratio of the nanowires will enhance the effects of photo-induced adsorption of gases like oxygen on the nanowire surface. Chemical treatments which etch the surface oxide can remove this surface layer as shown in Figure 16, restoring the PL response to its original spectrum. The nanowires that were damaged by laser beam were first examined by SEM and its chemical composition was determined by EDXS. It was found 


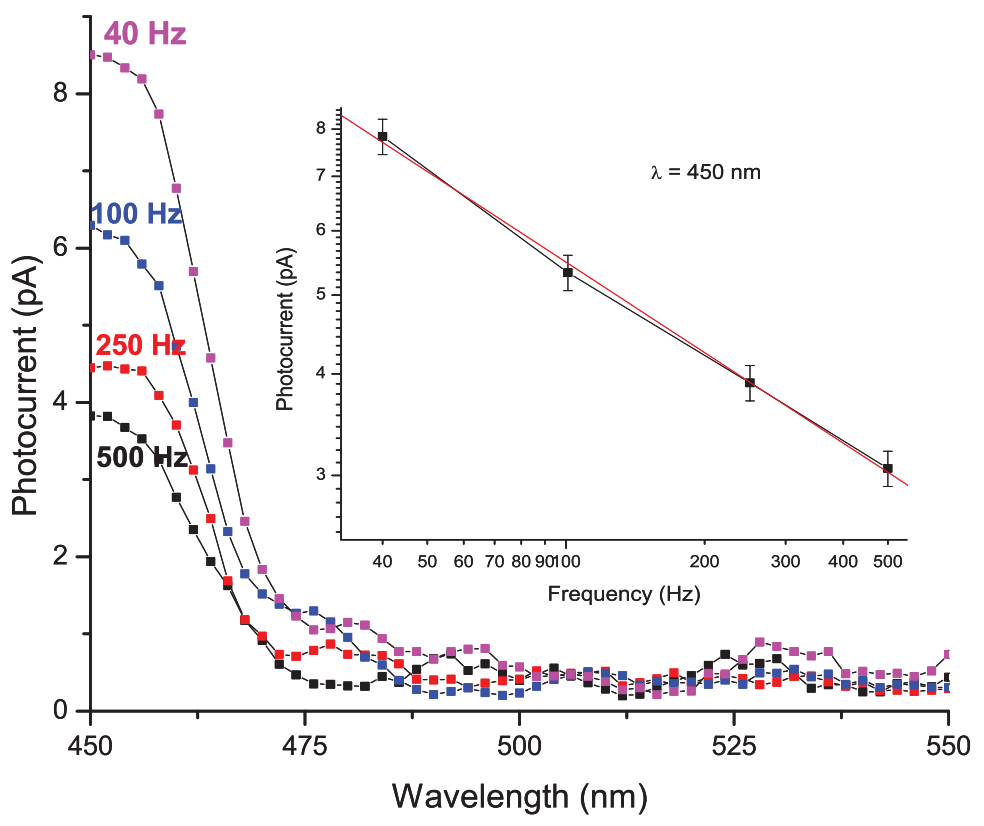

Fig. 14. Frequency dependence of photocurrent for the ZnSe nanowire array.

that the surface of the nanowire appeared roughened after exposure to the laser beam and also there was a higher concentration of oxygen detected at the surface. Hence the nanowires were treated with a solution of $\mathrm{H}_{2} \mathrm{SO}_{4}: \mathrm{H}_{2} \mathrm{O}_{2}: \mathrm{H}_{2} \mathrm{O}$ in the proportion 1:25:1 to etch off the surface oxide layer and restore the intensity of the NBE emission. At this stage, it was not certain if laser induced photo-oxidation was the only modification to the surface of the nanowire after it was exposed to laser irradiation. There is the possibility of laser induced formation of IPDs in the bulk as well as in the surface layer of the nanowire. In order to confirm the dominating phenomenon responsible for the quenching of the NBE emission, the nanowires were exposed to the same intensity of laser radiation, but in an inert atmosphere to prevent photo-induced oxidation. It was found that there was no quenching of the NBE emission; thus confirming that surface photo-oxidation of the nanowires was the dominating phenomena responsible for NBE emission quenching.

\section{Scanning photocurrent microscopy on nanowires}

Scanning photocurrent microscopy (SPCM) is a technique that has been used successfully to quantify the intrinsic properties of nanowires such as its mobility and ascertain the carrier transport mechanisms in nanowires. It has also been used to determine the effect of surface passivation schemes on charge transport in semiconductor nanowires ( $\mathrm{Gu}$ et al., 2006). A near-field scanning optical microscope (NSOM) is used to map the local photocurrent in individual nanowires contacted by electrodes and the response of the nanowires to local 


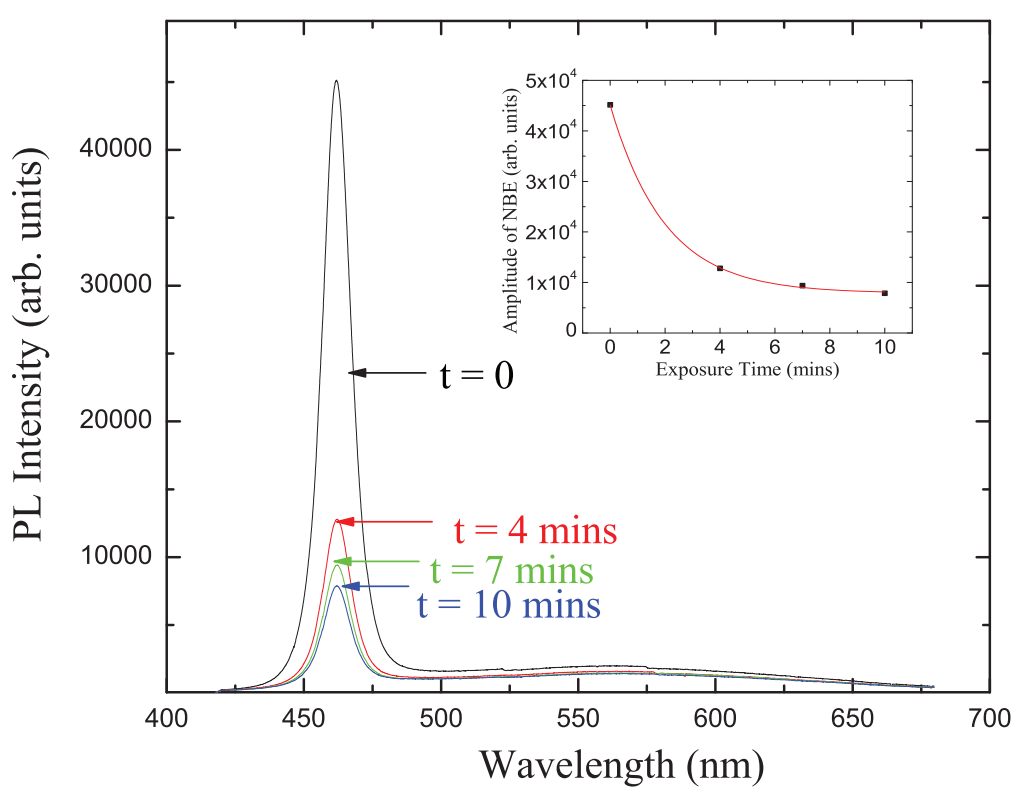

Fig. 15. Comparison of the room temperature PL spectra of ZnSe nanowires after laser illumination for 0 minutes; 4 minutes; 7 minutes; and 10 minutes. The inset shows the change in intensity with laser exposure time.

excitation is studied. Analysis of spatial variation and bias dependence on photocurrent enables determination of photocarrier transport and collection mechanisms. The resulting photocurrent image provides a two-dimensional map of the photocurrent versus NSOM tip position for a fixed electrode bias, as is reported by Y. Gu et al., (Gu et al., 2006). Analysis of the photocurrent profiles at different bias conditions can be used to determine the nature of electron and hole transport in the nanowire as well as a determination of its intrinsic properties.

\subsection{Surface passivation of nanowires}

The chemical and electronic stability of nanowire surfaces is important for its technological advancement. There are several strategies available for nanowire surface modification. Surface passivation can be achieved by the growth of an inorganic shell around the nanowire core or through organic functionalization. Several groups (Chang et al., 2006; Spencer et al., 2009) have reported on surface passivation achieved by the growth of $\mathrm{SiO}_{2}$ or $\mathrm{Si}_{3} \mathrm{~N}_{4}$ around a nanowire core made of either $\mathrm{ZnO}$ or $\mathrm{ZnSe}$. In the case of $\mathrm{ZnO}$, coated with $\mathrm{SiO}_{2}$, the electrical properties were found to be influenced by the chemisorption of oxygen on the nanowire surface. Oxygen molecules absorbed at the defect sites of $\mathrm{ZnO}$ nanowires act as electron acceptors to form $\mathrm{O}_{2}^{-}$ions, which then deplete the surface electron states and reduces channel 


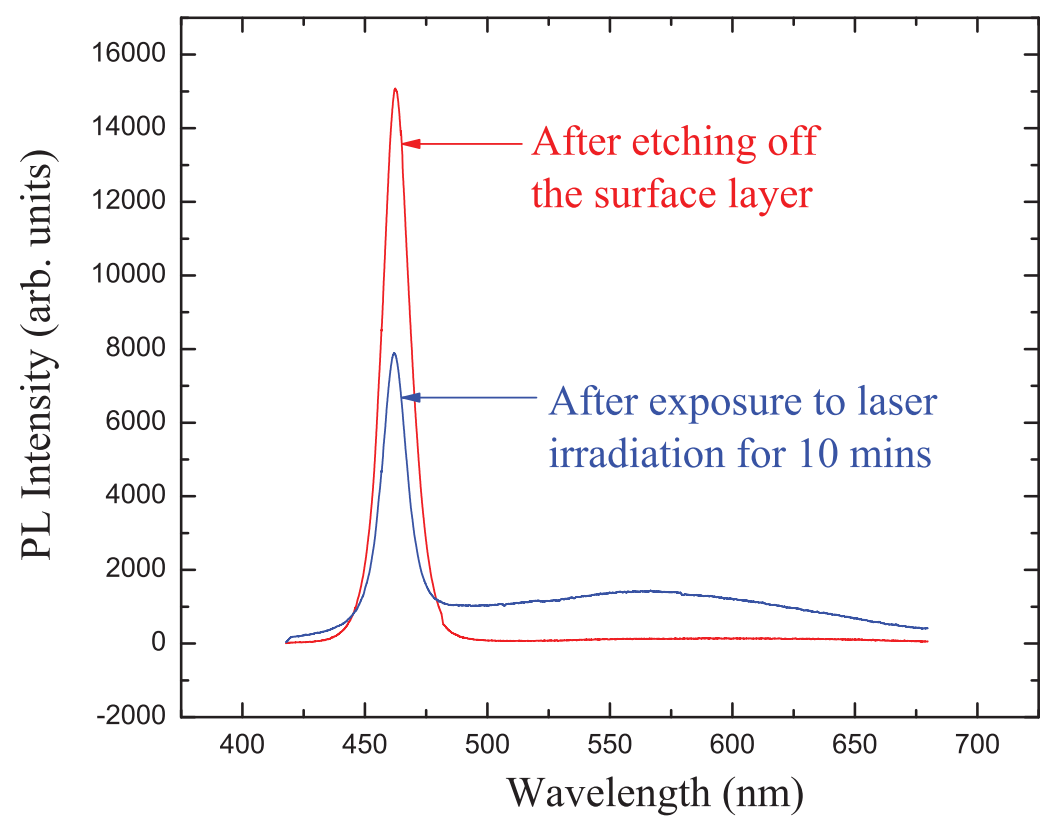

Fig. 16. Influence of etching on the PL intensity of ZnSe nanowires: After exposure to laser irradiation for 10 minutes (blue), and after etching (red).

conductivity. Hence, $\mathrm{ZnO}$ nanowire surfaces are passivated with a bi-layer of $\mathrm{SiO}_{2} / \mathrm{Si}_{3} \mathrm{~N}_{4}$, deposited by PECVD techniques serves as an effective passivating layer. Nanowire surfaces can also be passivated by Sulfur, in which case the nanowires are first etched using dilute HF acid, followed by temperature and time controlled treatment with $\left(\mathrm{NH}_{4}\right)_{2} \mathrm{~S}$ aqueous solution. Chloride- and hydride-terminated nanowires can similarly be produced by immersion in aqueous $\mathrm{HCl}$ or $\mathrm{HF}$ acid respectively.

\section{Conclusion}

Native point defects are always present in semiconductors and they notably affect the electrical and optical properties of the host material. The type and concentration of such defects in nanowires depend on the growth conditions. The optical and electronic properties of nanowires are defined to a large extent by these intrinsic point defects and a high density of defects is detrimental to the development of nanowires for device applications. The localized electronic states related to these defects degrades the characteristics of the material by acting as scattering or recombination centers for the charge carriers. Hence a detailed knowledge of the formation and nature of the defects is mandatory for the development of devices in high performance applications. There are several techniques for characterizing defects which 
includes structural, optical and electronic characterization. This chapter presents a brief review of the various techniques used in identifying and quantifying defect concentrations in semiconductor nanowires with special emphasis on ZnSe nanowires.

\section{References}

Chan, S. K., Cai, Y., Wang, N. \& Sou, I. K. (2006). Control of growth orientation for epitaxially grown znse nanowires, Applied Physics Letters 88: 013108:1-3.

Chang, P. C., Fan, Z., Chien, C., Stichtenoth, D., Ronning, C. \& Lu, J. (2006). High performance zno nanowire field effect transistors, Applied Physics Letters 89(13): 133113:1-3.

Gool, W. V. (1966). Principles of Defect Chemistry of Crystalline Solids, Arkin Publishing, Academic Press, New York.

Gu, Y., Romankiewicz, J. P., David, J. K., Lensch, J. L. \& Lauhon, L. J. (2006). Quantitative measurement of the electron and hole mobility lifetime products in semiconductor nanowires, Nano Letters 6(5): 948 - 952.

Laks, D. B., de Walle, C. G. V., Neumark, G. F., Blochl, P. E. \& Pantelides, S. T. (1992). Native defects and self compensation in znse, Physical Review B 45(19): 10965Ü10978.

Mal, S., Nori, S., Jin, C., Narayan, J., Nellutla, S., Smirnov, A. I. \& Prater, J. T. (2010). Reversible room temperature ferromagnetism in undoped zinc oxide: Correlation between defects and physical properties, Journal of Applied Physics 108(7): 073510:1-10.

Martinez-Canton, A., Garcia-Rocha, M., Hernandez-Calderon, I. \& Ortega-Martinez, R. (2005). Thermal quenching of the self-activated band of znse:cl thin films grown by molecular beam epitaxy, Microelectronics Journal 36(3-6): 527-530.

Othonos, A., Lioudakis, E., Philipose, U. \& Ruda, H. E. (2007). Ultrafast carrier dynamics in band edge and broad deep defect emission znse nanowires, Applied Physics Letters 91(24): 241113:1-3.

Philipose, U., P.Sun, Xu, T., Ruda, H. E., Yang, L. \& Kavanagh, K. L. (2007). Structure and photoluminescence of znse nanostructures fabricated by vapor phase growth, Journal Of Applied Physics 101: 014326:1-5.

Philipose, U., Ruda, H. E., Shik, A., de Souza, C. F. \& Sun, P. (2006). Conductivity and photoconductivity in undoped znse nanowire array, Journal of Applied Physics 99(6): 066106:1-3.

Philipose, U., Yang, S., Xu, T. \& Ruda, H. E. (2007). Origin of the red luminescence band in photoluminescence spectra of znse nanowires, Applied Physics Letters 90: 063103:1-3.

Potzger, K. \& Zhou, S. (2009). Non dms related ferromagnetism in transition metal doped zinc oxide, Physics Status Solidi B 246(6): 1147-1167.

Reshchikov, M. A. \& Morkoc, H. (2005). Luminescence properties of defects in gan, Journal of Applied Physics 97(6): 061301:1-95.

Rong, F. C., Barry, W. A., Donegan, J. F. \& G.D.Watkins (1996). Vacancies, interstitials, and close frenkel pairs on the zinc sublattice of znse, Physical Review B 54(11): 7779-7788.

Spencer, T. L., Cisek, R., Barzda, V., Philipose, U., Ruda, H. E. \& Shik, A. (2009). Orientation dependent nonlinear optical effects in znse nanowires, Applied Physics Letters 94: 233119:1-3.

Watkins, G. D. (1976). Radiation effects in semiconductors, 31: 95.

Zacks, E. \& Halperin, A. (1972). Dependence of the peak energy of the pair-photoluminescence band on excitation intensity, Physical Review B 6(8): 3072-3075. 


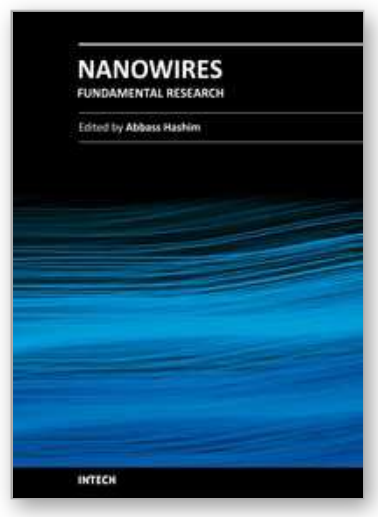

\author{
Nanowires - Fundamental Research \\ Edited by Dr. Abbass Hashim
}

ISBN 978-953-307-327-9

Hard cover, 552 pages

Publisher InTech

Published online 19, July, 2011

Published in print edition July, 2011

Understanding and building up the foundation of nanowire concept is a high requirement and a bridge to new technologies. Any attempt in such direction is considered as one step forward in the challenge of advanced nanotechnology. In the last few years, InTech scientific publisher has been taking the initiative of helping worldwide scientists to share and improve the methods and the nanowire technology. This book is one of InTechâ $€^{\mathrm{TM}_{\mathrm{S}}}$ attempts to contribute to the promotion of this technology.

\title{
How to reference
}

In order to correctly reference this scholarly work, feel free to copy and paste the following:

Usha Philipose (2011). Role of Intrinsic Defects in Nanowires, Nanowires - Fundamental Research, Dr. Abbass Hashim (Ed.), ISBN: 978-953-307-327-9, InTech, Available from: http://www.intechopen.com/books/nanowiresfundamental-research/role-of-intrinsic-defects-in-nanowires

\section{INTECH}

open science | open minds

\author{
InTech Europe \\ University Campus STeP Ri \\ Slavka Krautzeka 83/A \\ 51000 Rijeka, Croatia \\ Phone: +385 (51) 770447 \\ Fax: +385 (51) 686166 \\ www.intechopen.com
}

\author{
InTech China \\ Unit 405, Office Block, Hotel Equatorial Shanghai \\ No.65, Yan An Road (West), Shanghai, 200040, China \\ 中国上海市延安西路65号上海国际贵都大饭店办公楼 405 单元 \\ Phone: +86-21-62489820 \\ Fax: $+86-21-62489821$
}


(C) 2011 The Author(s). Licensee IntechOpen. This chapter is distributed under the terms of the Creative Commons Attribution-NonCommercialShareAlike-3.0 License, which permits use, distribution and reproduction for non-commercial purposes, provided the original is properly cited and derivative works building on this content are distributed under the same license. 